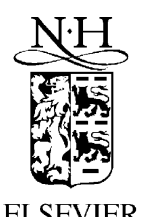

Applied Numerical Mathematics 33 (2000) 167-173

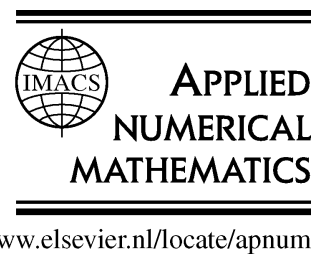

\title{
Pseudospectral solutions for steady motion of a viscous fluid inside a circular boundary
}

\author{
Weizhang Huang a , Tao Tang ${ }^{b, *}$ \\ a Department of Mathematics, University of Kansas, Lawrence, KS, USA \\ $\mathrm{b}$ Department of Mathematics, Hong Kong Baptist University, Hong Kong
}

\begin{abstract}
Numerical solutions are presented for steady two-dimensional motion within a circular cylinder generated by fluid injecting radially over one small arc and ejecting radially over another arc. These solutions are based on a mixed finite-difference pseudospectral method. Previous calculations were able to obtain convergent results only for a range of Reynolds numbers from $R e=0$ to $R e=20$. The main object of this study is to extend the Reynolds number range for reliable solution, particularly with regard to the flow patterns, based on a pseudospectral approach. @ 2000 IMACS. Published by Elsevier Science B.V. All rights reserved.
\end{abstract}

Keywords: Spectral method; Navier-Stokes; Circular geometry

\section{Introduction}

There are many differences between the external and the internal problems. For example, no solution of the external problem exists at zero Reynolds number (Stokes' paradox), but this is not true of the interior problem. These internal flows may occur through the rotation of part (or all) of the cylinder wall (moving-wall problem) or fluid entering and leaving the cylinder normal to the wall (inflow-outflow problem). These problems are of interest physically as they are representative of two distinct types of motion which occur frequently in practice (see Mills [10] and the references therein). They were also investigated theoretically and numerically by several researchers (see, e.g., $[1,3,9,11])$.

The motion inside a circular geometry is also a good model problem for testing numerical methods. It involves non-smooth boundary conditions, co-ordinate singularity (pole conditions), and stabilization for large Reynolds numbers. In spite of several numerical methods and computations for the interior flow problems, accurate results have been obtained only for small Reynolds number $(\operatorname{Re} \leqslant 25)$. The problem under consideration in this work is the inflow-outflow problem. The first attempt at this fluid motion was made by Rayleigh [11] who considered only slow motion. There have been also several approaches for

\footnotetext{
* Corresponding author. E-mail: ttang@math.hkbu.edu.hk
} 
solving the inflow-outflow problem numerically $[3,4,10,12]$, which all employed sequential iterative procedures. In using the iterative methods, difficulties arise in determining boundary values of the vorticity. In $[3,4,10,12]$, the boundary vorticity has been determined by applying a constraint condition derived from some integral representations. Convergent solutions have been obtained for small Reynolds numbers only. In this work, we will employ Newton's method rather than iterative methods in solving the discretized system, which avoids determining boundary values of the vorticity. The direct use of Newton's method on two-dimensional numerical approximations have been proved very successful (e.g., see [5]).

The main disadvantage in using Newton's method is that it requires large amount of computing time and computer memory. This had led many researchers to examine carefully the spectral method as a useful means for obtaining accurate solutions at a reasonably small number of a grid points. With spectral methods the relatively coarse grids allow very time- and memory-effective calculations. Although solutions to the present problems are nonsmooth, our numerical experiments suggest that spectral convergence in the radial direction can be obtained. Therefore, we will use a finite-difference approximation in the transverse direction and a pseudospectral method in the radial direction. Using this approach we are able to obtain accurate numerical solutions for high Reynolds numbers with practical computing time and computer memory.

\section{Mathematical formulation}

We shall consider the formulation for the general case of the inflow-outflow problem within the circular boundary as shown in Fig. 1. Two specific cases are considered numerically, namely the case of symmetrical flow with $\alpha=0$ and $\varepsilon=\pi / 30$ and the asymmetrical flow with $\alpha=\pi / 8$ and $\varepsilon=\pi / 32$. In this problem, the fluid is injected normally into the circle over an arc $\mathrm{CD}$ of length $2 \varepsilon$ and flows out at $\theta=\alpha$ over the arc $\mathrm{AB}$ of the same length as $\mathrm{CD}$. The radius $a$ of the circle, the velocity $U$ and half the flow $a \varepsilon U$ across the arc $\mathrm{CD}$ (or $\mathrm{AB}$ ) are used to make the variables dimensionless.

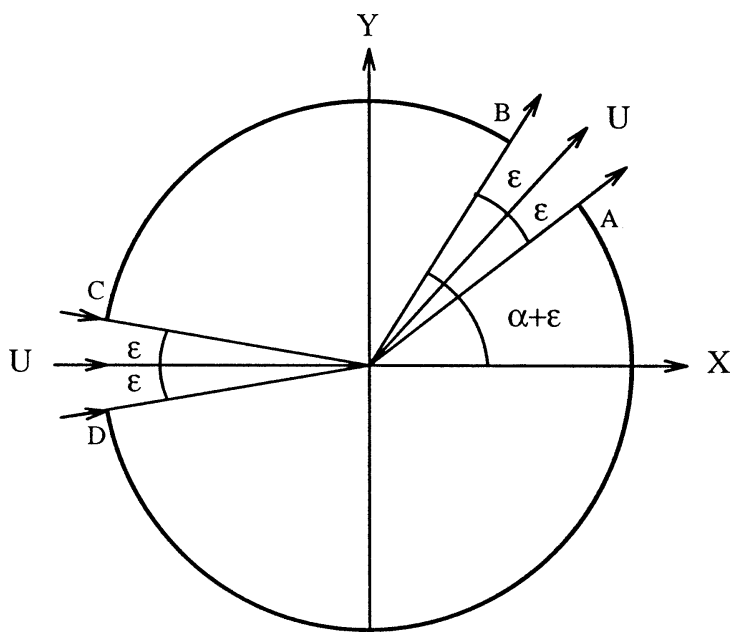

Fig. 1. Inflow-outflow problem. 
Taking plane polar coordinates $(r, \theta)$, the dimensionless radial and transverse velocity components $\left(v_{r}, v_{\theta}\right)$ and scalar vorticity $\zeta$ are defined by

$$
v_{r}=\frac{1}{r} \frac{\partial \psi}{\partial \theta}, \quad v_{\theta}=-\frac{\partial \psi}{\partial r}, \quad \zeta=\frac{1}{r} \frac{\partial v_{r}}{\partial \theta}-\frac{\partial v_{\theta}}{\partial r}-\frac{v_{\theta}}{r},
$$

where $\psi$ is the streamfunction. From (1) and the Navier-Stokes momentum equations, the governing equations for $\psi$ and $\zeta$ are well-known to be

$$
\Delta \psi=\zeta, \quad \Delta \zeta=\frac{\operatorname{Re}}{r}\left(\frac{\partial \psi}{\partial \theta} \frac{\partial \zeta}{\partial r}-\frac{\partial \psi}{\partial r} \frac{\partial \zeta}{\partial \theta}\right)
$$

where $\operatorname{Re}=U a \varepsilon / v$ is the Reynolds number of the motion, $v$ is the kinematic viscosity coefficient of the fluid and $\Delta=\partial_{r r}+r^{-1} \partial_{r}+r^{-2} \partial_{\theta \theta}$.

The boundary conditions for the present problem (see Fig. 1) are that, at $r=1$,

$$
\begin{aligned}
& \psi=f(\theta)= \begin{cases}(\theta-\alpha) / \varepsilon, & \text { for } \alpha-\varepsilon \leqslant \theta \leqslant \alpha+\varepsilon, \\
1, & \text { for } \alpha+\varepsilon \leqslant \theta \leqslant \pi-\varepsilon, \\
(\pi-\theta) / \varepsilon, & \text { for } \pi-\varepsilon \leqslant \theta \leqslant \pi+\varepsilon, \\
-1, & \text { for } \pi+\varepsilon \leqslant \theta \leqslant 2 \pi+\alpha-\varepsilon,\end{cases} \\
& \frac{\partial \psi}{\partial r}=0 \text { for } 0 \leqslant \theta \leqslant 2 \pi .
\end{aligned}
$$

The governing equations (2) are singular at $r=0$. Some special treatment at this point is required for finite difference or pseudospectral approximations. It can be shown that spectral accuracy is obtained if the (standard) pseudospectral method is applied to such coordinate-singular problems with suitable pole conditions (e.g., see [2,6]). It is assumed that at $r=0$ the stream function and vorticity are smooth and unique (single valued). This, together with the Navier-Stokes equations (2) and Taylor expansion, yield:

$$
\frac{\partial^{2} \psi}{\partial \theta^{2}}(0, \theta)=0, \quad \int_{0}^{2 \pi} \frac{\partial \psi}{\partial r}(0, \tilde{\theta}) \mathrm{d} \widetilde{\theta}=0, \quad \frac{\partial^{2} \zeta}{\partial \theta^{2}}(0, \theta)=0, \quad \int_{0}^{2 \pi} \frac{\partial \zeta}{\partial r}(0, \tilde{\theta}) \mathrm{d} \widetilde{\theta}=0 .
$$

They are used as numerical boundary conditions at $r=0$. For $\alpha=0$ these conditions can be significantly simplified for the case of symmetrical flow due to the symmetry. In this case, we have

$$
\psi(0, \theta)=0 \text { and } \zeta(0, \theta)=0 \text { for } 0 \leqslant \theta \leqslant 2 \pi .
$$

\section{Numerical methods}

We use central finite differences in the $\theta$-direction and a pseudospectral (Legendre collocation) method in the $r$-direction. Let $\rho_{i}, 0 \leqslant i \leqslant N_{r}$ be a set of Legendre-Gauss-Lobatto points on [-1, 1], where $N_{r}$ is a positive integer. We define transformed collocation points in the $r$-direction as $r_{i}=\left(1+\rho_{i}\right) / 2$. In the $\theta$-direction grid points are defined as $\theta_{j}=j h_{\theta}, h_{\theta}=2 \pi / N_{\theta}, 0 \leqslant j \leqslant N_{\theta}$. Denote the approximations of $\psi\left(r_{i}, \theta_{j}\right)$ and $\zeta\left(r_{i}, \theta_{j}\right)$ by $\psi_{i, j}$ and $\zeta_{i, j}$, respectively, and define

$$
\psi_{j}(r)=\sum_{i=0}^{N_{r}} \psi_{i, j} L_{i}(r), \quad \zeta_{j}(r)=\sum_{i=0}^{N_{r}} \zeta_{i, j} L_{i}(r), \quad 0 \leqslant j \leqslant N_{\theta}
$$


where $L_{i}(r)$ are the Lagrange basis functions associated with $\left\{r_{k}\right\}_{k=0}^{N_{r}}$. Then discretizing the governing equations (2) by using central differences in the $\theta$-direction and the pseudospectral method in the $r$-direction at the interior grid points gives: at $r=r_{i}$,

$$
\begin{aligned}
& \left(\frac{\mathrm{d}^{2}}{\mathrm{~d} r^{2}}+\frac{1}{r} \frac{\mathrm{d}}{\mathrm{d} r}\right) \psi_{j}(r)+\frac{\psi_{j+1}(r)-2 \psi_{j}(r)+\psi_{j-1}(r)}{r^{2} h_{\theta}^{2}}=\zeta_{j}(r), \\
& \left(\frac{\mathrm{d}^{2}}{\mathrm{~d} r^{2}}+\frac{1}{r} \frac{\mathrm{d}}{\mathrm{d} r}\right) \zeta_{j}(r)+\frac{\zeta_{j+1}(r)-2 \zeta_{j}(r)+\zeta_{j-1}(r)}{r^{2} h_{\theta}^{2}} \\
& =\frac{\operatorname{Re}}{r}\left[\frac{\psi_{j+1}(r)-\psi_{j-1}(r)}{2 h_{\theta}} \frac{\mathrm{d} \zeta_{j}}{\mathrm{~d} r}(r)-\frac{\mathrm{d} \psi_{j}}{\mathrm{~d} r}(r) \frac{\zeta_{j+1}(r)-\zeta_{j-1}(r)}{2 h_{\theta}}\right],
\end{aligned}
$$

for $1 \leqslant i \leqslant N_{r}-1,0 \leqslant j \leqslant N_{\theta}-1$.

For high Reynolds numbers it is often required for numerical schemes to have some type of upwind features in order to stabilize the computation. Here we employ an upwind treatment for the pseudospectral approximation in the $r$-direction. This technique has been used successfully by Huang and Sloan [7] for solving a class of singular perturbation problems.

Define the approximation of the velocity component $v_{r}$ as $\left(v_{r}\right)_{i, j}=\left(\psi_{j+1}\left(r_{i}\right)-\psi_{j-1}\left(r_{i}\right)\right) / 2 r_{i} h_{\theta}$ and let

$$
\widehat{\zeta}_{j}(r)=\sum_{i=0}^{N_{r}-1} \zeta_{i, j} \widehat{L}_{i}(r), \quad \check{\zeta}_{j}(r)=\sum_{i=1}^{N_{r}} \zeta_{i, j} \check{L}_{i}(r), \quad 0 \leqslant j \leqslant N_{\theta},
$$

where $\widehat{L}_{i}(r)$ are the Lagrange basis functions associated with $\left\{r_{k}\right\}_{k=0}^{N_{r}-1}, \check{L}_{i}(r)$ are associated with $\left\{r_{k}\right\}_{k=1}^{N_{r}}$. Then replace the term $\left(\mathrm{d} \zeta_{j} / \mathrm{d} r\right)\left(r_{i}\right)$ on the right-hand side of Eq. (8) by

$$
\frac{\mathrm{d} \zeta_{j}}{\mathrm{~d} r}\left(r_{i}\right) \Rightarrow \begin{cases}\frac{\mathrm{d} \widehat{\zeta}_{j}}{\mathrm{~d} r}\left(r_{i}\right), & \text { if }\left(v_{r}\right)_{i, j} \geqslant 0, \\ \frac{\mathrm{d} \check{\zeta}_{j}}{\mathrm{~d} r}\left(r_{i}\right), & \text { if }\left(v_{r}\right)_{i, j}<0 .\end{cases}
$$

This treatment has been used in all calculations presented in this paper and it is found that this technique stabilizes the computation. It is especially useful for the case of asymmetrical flow with high Reynolds numbers.

The system consisting of (7), (8) and related boundary conditions is nonlinear and is solved by Newton's method with continuation in Re.

\section{Numerical results}

We shall investigate two typical cases of the inflow-outflow problem as described in Fig. 1, the symmetrical flow with $(\alpha, \varepsilon)=(0, \pi / 30)$ and the asymmetrical case with $(\alpha, \varepsilon)=(\pi / 8, \pi / 32)$. We first consider the symmetrical flow problem which has been investigated numerically in $[3,4,12]$.

To examine the accuracy of the numerical method described in the previous section and its capability of handling the coordinate singularity at $r=0$, numerical experiments have been carried out for the flow with $\operatorname{Re}=0$ where the analytical expression of the solution is available [10]. First we fix $N_{\theta}$ and consider the accuracy of the method for a couple of values of $N_{r}$. A relatively large $N_{\theta}\left(N_{\theta}=140\right)$ is chosen so 

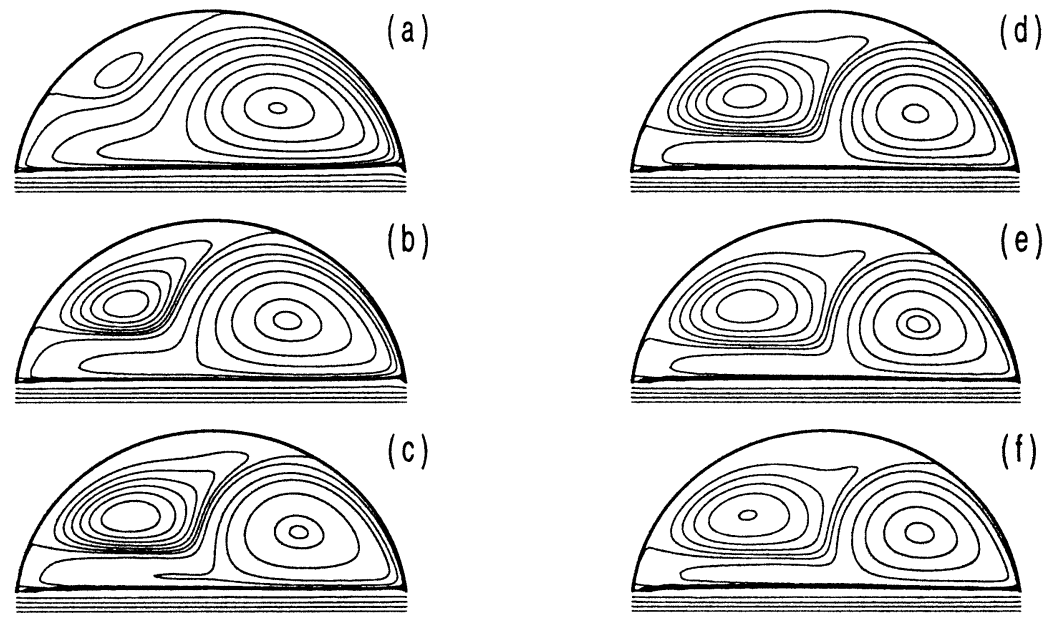

Fig. 2. Streamlines for the symmetrical flow with (a) $\operatorname{Re}=100$, (b) $\operatorname{Re}=200$, (c) $\operatorname{Re}=400$, (d) $\operatorname{Re}=600$, (e) $\operatorname{Re}=800$ and (f) $\operatorname{Re}=1000$.

Table 1

Values for $\psi, \zeta$ and coordinates at the centers of the primary vortex (with subscript 1) and secondary vortex (with subscript 2)

\begin{tabular}{rcrcccccc}
\hline $\operatorname{Re}$ & $\psi_{1}$ & $\zeta_{1}$ & $x_{1}$ & $y_{1}$ & $\psi_{2}$ & $\zeta_{2}$ & $x_{2}$ & $y_{2}$ \\
\hline 100 & 1.554 & -12.306 & 0.337 & 0.461 & 0.993 & 1.401 & -0.479 & 0.653 \\
200 & 1.449 & -10.739 & 0.384 & 0.452 & 0.971 & 2.074 & -0.440 & 0.537 \\
400 & 1.324 & -8.824 & 0.441 & 0.438 & 0.965 & 1.731 & -0.429 & 0.522 \\
800 & 1.214 & -6.467 & 0.477 & 0.431 & 0.972 & 1.153 & -0.408 & 0.529 \\
1000 & 1.186 & -5.682 & 0.481 & 0.432 & 0.975 & 0.984 & -0.402 & 0.530 \\
\hline
\end{tabular}

that the discretization error in the $\theta$-direction is sufficiently small. It is observed that spectral accuracy is indeed achieved and the numerical results with a few collocation points are fairly accurate. Next we fix $N_{r}=24$ and vary $N_{\theta}$. As expected the convergence order of the central difference scheme is about 1.8 which less than two due to the nonsmoothness of the boundary conditions at $r=1$. Numerical results also suggest that the grid sizes in the $\theta$-direction should be chosen such that no grid points coincide with the sharp edges. When the sharp edges are grid points the numerical errors become larger.

The streamlines for the cases of $2.5 \leqslant \mathrm{Re} \leqslant 20$ obtained with $N_{r}=24, N_{\theta}=140$ are in excellent agreement with the published results. The streamlines obtained with grid $N_{r}=40$ and $N_{\theta}=160$ for higher Reynolds numbers $100 \leqslant \mathrm{Re} \leqslant 1000$ are shown in Fig. 2. It is observed that a secondary vortex appears around $\mathrm{Re}=100$. The secondary vortex becomes stronger until about $\operatorname{Re}=400$ and is subsequently getting weaker as $R$ increases. This can also be seen from Table 1 where the values of the stream function, vorticity and coordinates at the primary and secondary vortex centers are listed. Table 1 also suggests that positions of the vortex centers tend to be independent of Re as the Reynolds number increases. 

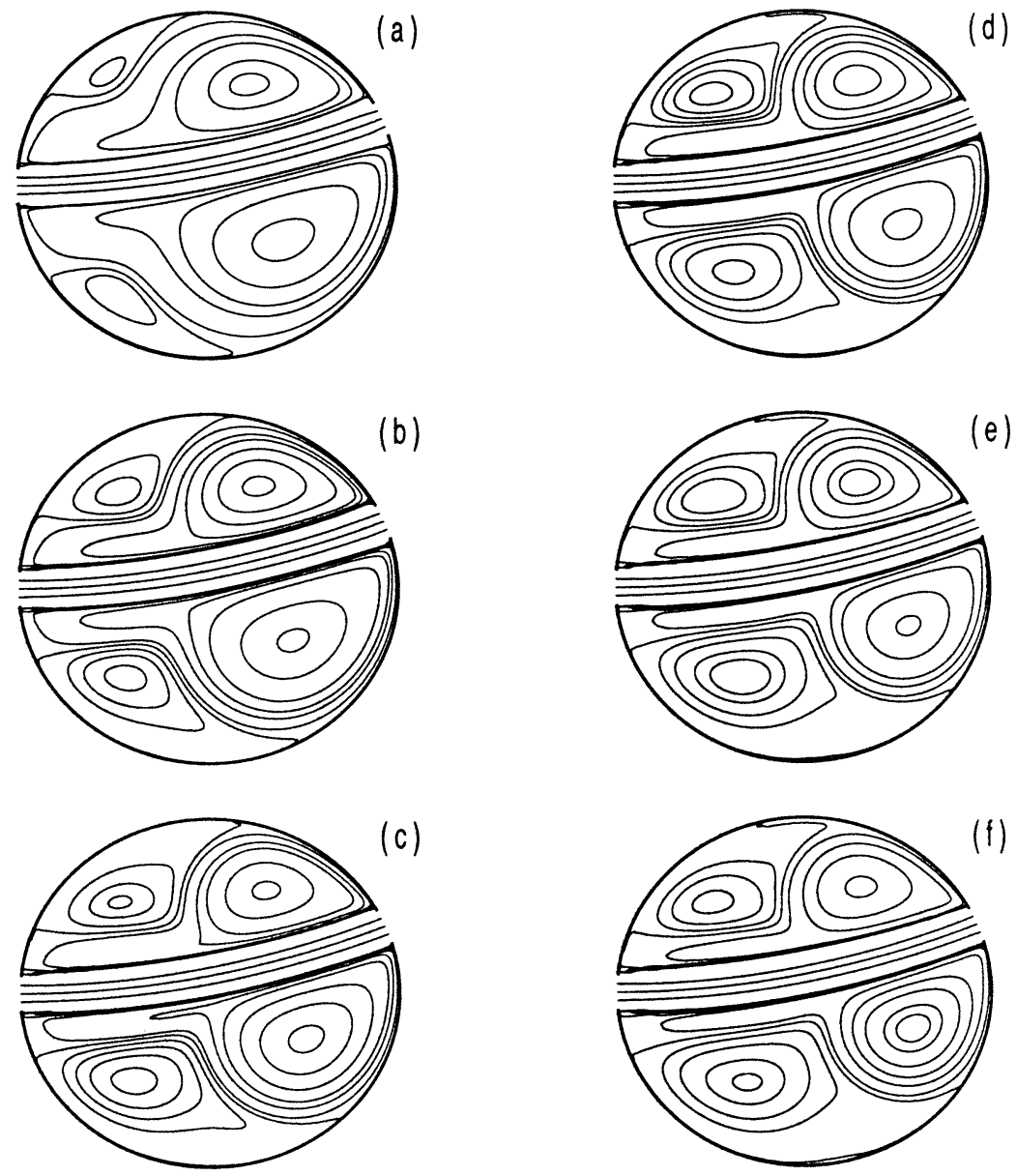

Fig. 3. Streamlines for the asymmetrical flow with (a) $\operatorname{Re}=100$, (b) $\operatorname{Re}=200$, (c) $\operatorname{Re}=400$, (d) $\operatorname{Re}=600$, (e) $\operatorname{Re}=800$ and (f) $\operatorname{Re}=1000$.

In order to ensure that $N_{\theta}=160$ is larger enough for higher Reynolds numbers, results for $\operatorname{Re}=200$ and 600 with different values of $N_{\theta}$ are computed. It is noticed that the changes from $N_{\theta}=160$ to 200 are very small.

Finally, we consider asymmetrical flow with $\alpha=\pi / 8$ and $\varepsilon=\pi / 32$. This problem has been investigated by Mills [10] and Dennis et al. [4] for $\mathrm{Re} \leqslant 20$. Again, our results for $\mathrm{Re} \leqslant 20$ are in good agreement with those given in [4]. They are also comparable with those presented by Mills [10] except for the case of $\operatorname{Re}=2.5$. In this case, our result consolidates the observation of Dennis et al. who found two regions of separation rather than one as shown by Mills.

The streamlines obtained with $\left(N_{r}, N_{\theta}\right)=(40,160)$ for $100 \leqslant \operatorname{Re} \leqslant 1000$ are shown in Fig. 3. The values of the stream function, vorticity and coordinates at the vortex centers are listed in Table 2 . It is observed that the flow patterns for large values of Re are almost independent of the Reynolds numbers.

The full spectral methods in both $r$ and $\theta$ directions, based on spectral element techniques, can be found in [8]. 
Table 2

Values for $\psi, \zeta$ and coordinates at the centers of the primary vortices (the upper one with subscript 11 and the lower with 12) and secondary vortices (the upper one with subscript 21 and the lower with 22) for the asymmetrical flow

\begin{tabular}{ccccccccc}
\hline $\operatorname{Re}$ & $\psi_{11}$ & $\zeta_{11}$ & $x_{11}$ & $y_{11}$ & $\psi_{12}$ & $\zeta_{12}$ & $x_{12}$ & $y_{12}$ \\
\hline 100 & 1.501 & -14.509 & 0.222 & 0.583 & -1.647 & 11.111 & 0.400 & -0.318 \\
200 & 1.364 & -12.288 & 0.265 & 0.586 & -1.532 & 9.763 & 0.446 & -0.299 \\
400 & 1.279 & -9.398 & 0.297 & 0.590 & -1.394 & 8.345 & 0.507 & -0.264 \\
800 & 1.182 & -6.370 & 0.304 & 0.596 & -1.266 & 6.710 & 0.564 & -0.226 \\
1000 & 1.157 & -5.513 & 0.305 & 0.600 & -1.231 & 6.198 & 0.580 & -0.215 \\
\hline $\operatorname{Re}$ & $\psi_{21}$ & $\zeta_{21}$ & $x_{21}$ & $y_{21}$ & $\psi_{22}$ & $\zeta_{22}$ & $x_{22}$ & $y_{22}$ \\
\hline 100 & 0.993 & 1.609 & -0.520 & 0.646 & -0.991 & -1.399 & -0.473 & -0.624 \\
200 & 0.976 & 2.229 & -0.471 & 0.549 & -0.966 & -2.047 & -0.441 & -0.506 \\
400 & 0.974 & 1.765 & -0.475 & 0.524 & -0.957 & -1.697 & -0.406 & -0.502 \\
800 & 0.981 & 1.173 & -0.494 & 0.514 & -0.961 & -1.135 & -0.331 & -0.518 \\
1000 & 0.984 & 0.996 & -0.496 & 0.514 & -0.964 & -0.979 & -0.307 & -0.521 \\
\hline
\end{tabular}

\section{References}

[1] G.B. Batchelor, On steady laminar flow with closed streamlines at large Reynolds number, J. Fluid Mech. 1 (1956) 177-190.

[2] C. Canuto, M.Y. Hussaini, A. Quarteroni, T.A. Zang, Spectral Methods in Fluid Dynamics, Springer-Verlag, Berlin/Heidelberg, 1988.

[3] S.C.R. Dennis, in: Proc. 4th Int. Conf. on Numer. Methods in Fluid Dyn., Lecture Notes in Physics, Vol. 35 , Springer-Verlag, New York/Berlin, 1975, p. 138.

[4] S.C.R. Dennis, M. Ng, P. Nguyen, Numerical solution for the steady motion of a viscous fluid inside a circular boundary using integral conditions, J. Comput. Phys. 108 (1993) 142-152.

[5] B. Fornberg, Steady viscous flow past a sphere at high Reynolds numbers, J. Fluid Mech. 190 (1991) 471-489.

[6] W.-Z. Huang, D.M. Sloan, Pole condition for singular problems: the pseudospectral approximation, J. Comput. Phys. 107 (1993) 254-261.

[7] W.-Z. Huang, D.M. Sloan, A new pseudospectral method with upwind features, IMA J. Numer. Anal. 13 (1993) 413-430.

[8] A. Karageorghis, T. Tang, A spectral domain decomposition approach for steady Navier-Stokes problems in circular geometries, Comput. Fluids 25 (1996) 541-549.

[9] K. Kuwahara, I. Imai, Phys. Fluids Suppl. 12, II (1969) 94.

[10] R.D. Mills, Computing internal viscous flow problems for the circle by integral methods, J. Fluid Mech. 79 (1977) 609-624.

[11] L. Rayleigh, Phil. Mag. 5 (1893) 354.

[12] C.M. Wang, J.C. Wu, Numerical solution of steady Navier-Stokes problems using integral representations, AIAA J. 24 (1986) 1305-1312. 